\title{
The intractable problem of chronic disease
}

$\mathrm{H}$ ow we manage chronic disease has led to major changes in our health care systems. Hospitals have been downsized on the premise that chronic diseases should be managed in the community, to prevent them from becoming acute. To meet this need, Canada and most other western nations are gradually replacing single-practice family medicine with a range of team-based models for primary care. This shift is in turn transforming the way many medical schools recruit and train students. We can learn from the past to inform these changes.

The term "chronic disease" goes back to the ancient Greeks. It is related to time. In contrast to acute diseases, which either kill you or disappear quickly, chronic diseases are long and lingering. During the 18th and 19th centuries, chronic disease was frequently discussed as a single category by systemic thinkers, who postulated one constitutional cause for many conditions. William Cadogan, in his often-reprinted book of the 18th century, argued that all chronic diseases were the result of "indolence, intemperance and vexation." Samuel Hahnemann, the founder of homeopathy, thought that chronic diseases were the products of "miasms," dynamic noxious influences. The most important and oldest of these, psora, was responsible for numerous conditions. Hundreds of medical books on chronic disease were published during this period; clearly, these diseases played a large role both in people's lives and in physicians' practices.

During the 19th century, chronic disease lost its status as a recognizable condition. As medical thought became centred on disease entities defined by specific causes and organic lesions, the word "chronic" became an adjective applied to any disease or condition that persisted. The term "chronic disease" once again achieved prominence as a specific category in the early 20th century, but carrying an entirely new meaning. No longer just a set of lingering diseases and conditions, chronic disease became a major social problem that demanded collective social action. For several reasons, this development occurred primarily in the United States.

One compelling reason, which can be traced back to the early 19 th century, was the desire to get patients with chronic diseases out of hospitals. Patients in seemingly hopeless situations were taking up beds that, it was thought, could be put to better use. This belief intensified as hospitals increasingly focused on acute and curable diseases.

Transformed beliefs and attitudes also fuelled the perceived chronic disease crisis of the early 20th century. growth of large cities and the attendant huge increase in the concentration of chronically ill and disabled people who could not work and who could not be dealt with by inadequate and overstretched public welfare systems or private charity. Keeping people off the welfare rolls became an important rationale for prevention and cure.

Perhaps the most direct factor in producing this new concern was the apparent change in disease-related mortality. By the early decades of the 20th century, public health leaders in the US believed that although mortality rates were falling generally, those for certain diseases of adults, notably cancers, cardiovascular diseases and diabetes mellitus, seemed to be increasing rapidly. "Apparent" is the operative word here, because there were many reasons to doubt the statistics, as critics hastened to point out. The systematic

\section{Chronic disease became a major social problem that demanded collective social action.}

Fresh fears about the effects of "civilization" (sedentary urban life, overconsumption, alcohol abuse, overcrowding and, for eugenicists, unrestricted breeding) became widespread. This anxiety about collective degeneration spurred new concerns about chronic conditions, such as mental disability, that were viewed as emblematic of societal decline.

Simultaneously, a new sense of optimism and social responsibility made some reformers believe that previously hopeless conditions could be cured, ameliorated or controlled by scientific medicine and that everyone had the right to proper medical care. This view was largely a consequence of the collection of statistics in the US was only beginning, disease categories were changing rapidly, diagnostic methods were improving, specialists were now actively looking for diseases that had been long ignored, and general practitioners who filled out death certificates with little personal knowledge of the deceased were more prone than they had been previously to list such newly visible diseases as the cause of death. Most observers agreed that lower infant and child mortality rates undoubtedly increased the amount of adult illness, but not nearly enough to explain the alarming statistics. Nonetheless, these statistics combined with political considerations to create a social movement. 


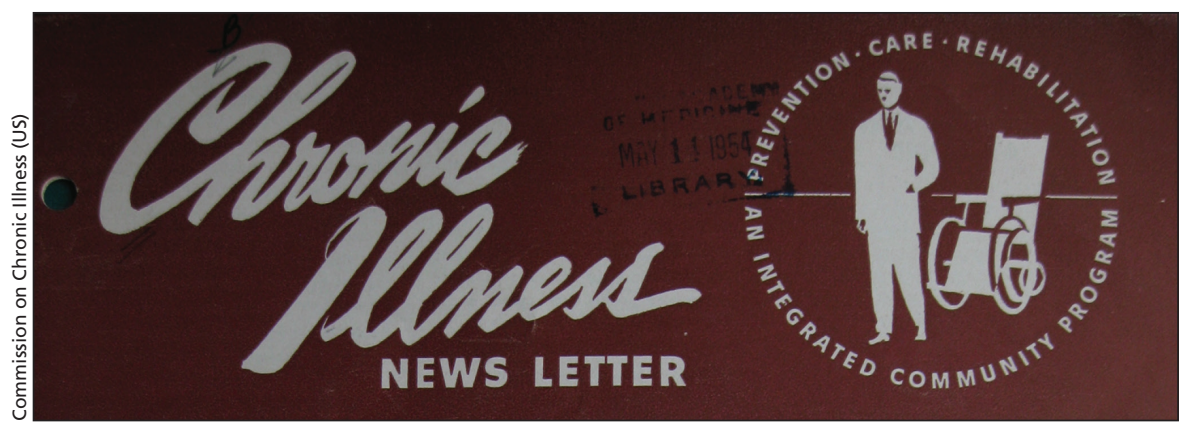

Figure 1: Banner for the newsletter of the US Commission on Chronic Illness (19491957).

During the 1930s and 1940s, the rising rate of chronic diseases was used to justify the creation of national health insurance in the US (an effort that failed). In subsequent decades, the need to confront chronic diseases became an organizing principle for US health care reform because, unlike the highly divisive issue of national health insurance, the issue of chronic disease evoked broad consensus that could direct policy. It led to the public-private Commission on Chronic Illness (19491957), which cast chronic disease as a major social problem (Figure 1).

Canadian doctors were deeply concerned about specific chronic illnesses, particularly cancers, in the 1930s and '40s, but there were no efforts to build health policy around chronic disease. In Canada, as in Europe, the prospects of universal health insurance left little room for an alternative framework targeting specific groups of patients. Certainly, the Canadian Sickness Survey of 1950-1951 counted "chronic disabilities" in about $3 \%$ of the population and noted that among the major types were heart disease, arthritis and rheumatism, and disorders of the nervous system. ${ }^{1}$ However, the major emphasis in early discussions of the survey was on household expenditures on health, ${ }^{2,3}$ and the survey can best be seen as part of the process of discussion and reflection that led first to the Hospital Insurance and Diagnostic Services Act (1957) and the Medical
Care Act (1966). However, K.C. Charron, director of health services in the Department of National Health and Welfare, published articles every few years warning that the prevention and control of chronic disease "is the great health challenge of the future."

In the decades that followed, the need to prevent and treat chronic conditions has become an ever-greater preoccupation in Canada and other developed countries. Most western nations have developed disease-specific control programs, as well as more general programs that target chronic diseases as a group. Lacking the wealth of the US, few nations have followed that country's experiments with multiphasic screening, the effort to locate a variety of diseases through low-cost and mechanized procedures or to introduce annual medical examinations. Most, however, have introduced some targeted screening (e.g., for breast cancer) and have organized public campaigns to reduce unhealthy behaviours like smoking, lack of exercise and excessive alcohol consumption. Epidemiology has provided the concept of risk factors that can be treated through behaviour change or medication or both.

Preoccupation with chronic diseases has increased over time, in part because of continuing epidemiologic change that has produced large aging populations, but also as a consequence of this very preoccupation. Some chronic diseases reflect traditional disease categories, but our criteria for defining and treating them as diseases (cancer in situ, mild diabetes) tend to expand, as does the propensity to overdiagnose. Furthermore, many new diseases are being created. Mildly high blood pressure, recognized a half-century ago as a risk factor for cardiovascular disease, is now treated as the disease hypertension. Behaviours once considered social problems have become medical conditions treated with medication (e.g., attention-deficit/hyperactivity disorder). Having certain genes is now considered a reason for breast removal surgery.

My point here is not to criticize there is enough criticism of "medicalization" out there already — but simply to point out that chronic disease is an ever-expanding category that is partly of our own making. That is one of the reasons it is so hard to confront effectively.

\section{George Weisz PhD}

Department of Social Studies of Medicine, McGill University, Montréal, Que.

\section{References}

1. Cameron GDW. The Canadian Sickness Survey 1950-51: its implications for the practising physician. Can Med Assoc J 1954;71:613-5.

2. Francis CL. Expenditure patterns from the Canadian Sickness Survey 1950-1951. Can J Public Health 1956;47:328-31.

3. Peart AFW. Canada's sickness survey; review of methods. Can J Public Health 1952;43:401-14.

4. Charron KC. Chronic disease in the Canadian Hospital Program. Can J Public Health 1957;48:405-12.

5. Charron KC. The magnitude of chronic disease in Canada. Can J Public Health 1961;52:273-84.

6. Charron KC. The problem of chronic disease within the overall framework of health care in Canada. J Am Geriatr Soc 1964;12:50-60.

This article has been peer reviewed.

CMAJ 2016. DOI:10.1503/cmaj.160354

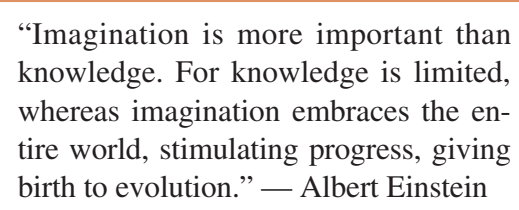

"Imagination is more important than knowledge. For knowledge is limited, birth to evolution." - Albert Einstein 